\title{
Вмј Global Health Addressing the information deficit in global health: lessons from a digital acute care platform in Sri Lanka
}

Abi Beane, ${ }^{1,2}$ Ambepitiyawaduge Pubudu De Silva, ${ }^{3}$ Priyantha Lakmini Athapattu, ${ }^{4}$ Saroj Jayasinghe, ${ }^{5}$ Anuja Unnathie Abayadeera, ${ }^{6}$ Mandika Wijerathne, ${ }^{6}$ Ishara Udayanga, ${ }^{1}$ Shriyananda Rathnayake, ${ }^{7}$ Arjen M Dondorp, ${ }^{2}$ Rashan Haniffa ${ }^{1,2}$

To cite: Beane A, De Silva AP, Athapattu PL, et al. Addressing the information deficit in global health: lessons from a digital acute care platform in Sri Lanka. BMJ Glob Health 2019;4:e001134. doi:10.1136/ bmjgh-2018-001134

Handling editor Soumitra Bhuyan

Received 22 August 2018 Revised 8 December 2018 Accepted 13 December 2018

Check for updates

(C) Author(s) (or their employer(s)) 2019. Re-use permitted under CC BY-NC. No commercial re-use. See rights and permissions. Published by BMJ.

${ }^{1}$ Network for Improving Critical Care Systems and Training,

Colombo, Sri Lanka

${ }^{2}$ Mahidol Oxford Tropical Research Unit, Bangkok, Thailand

${ }^{3}$ National Intensive Care Surveillance, Ministry of Health, Colombo, Sri Lanka

${ }^{4}$ Ministry of Health, Colombo,

Sri Lanka

${ }^{5}$ Department of Medicine,

Faculty of Medicine, University of Colombo, Colombo, Sri Lanka ${ }^{6}$ Department of Surgery, Faculty of Medicine, University of Colombo, Colombo, Sri Lanka

${ }^{7}$ Information and

Communication Technology Agency of Sri Lanka, Columbo, Sri Lanka

Correspondence to

Ms Abi Beane; abi@nicslk.com

\section{ABSTRACT}

Lack of investment in low-income and middle-income countries (LMICs) in systems capturing continuous information regarding care of the acutely unwell patient is hindering global efforts to address inequalities, both at facility and national level. Furthermore, this of lack of data is disempowering frontline staff and those seeking to support them, from progressing setting-relevant research and quality improvement. In contrast to high-income country (HIC) settings, where electronic surveillance has boosted the capability of governments, clinicians and researchers to engage in service-wide healthcare evaluation, healthcare information in resource-limited settings remains almost exclusively paper based. In this practice paper, we describe the efforts of a collaboration of clinicians, administrators, researchers and healthcare informaticians working in South Asia, in addressing the inequality in access to patient information in acute care. Harnessing a clinician-led collaborative approach to design and evaluation, we have implemented a national acute care information platform in Sri Lanka that is tailored to priorities of frontline staff. Iterative adaptation has ensured the platform has the flexibility to integrate with legacy paper systems, support junior team members in advocating for acutely unwell patients and has made information captured accessible to diverse stakeholders to improve service delivery. The same platform is now empowering clinicians to participate in international research and drive forwards improvements in care. During this journey, we have also gained insights on how to overcome well-described barriers to implementation of digital information tools in LMIC. We anticipate that this north-south collaborative approach to addressing the challenges of health system implementation in acute care may provide learning and inspiration to other partnerships seeking to engage in similar work.

\section{INTRODUCTION}

Disparity in quality of care is increasingly recognised as important causes of excess mortality and morbidity in acute healthcare internationally. In South Asia, services essential to the management of acute conditionssurgical, medical and critical care-are
Summary box

- Continuous acute care surveillance using digital platforms in South Asia is feasible.

- Clinician-led co-development and evaluation can facilitate adoption of digital platforms into existing health systems.

- Information captured through the digital platform enables evaluation of existing patient care at facility and national level, facilitates participation of low-income and middle-income countries in internationa research and empowers clinicians to identify priorities for care improvement.

becoming increasingly available in the region, given the rising burden of non-communicable disease. Data from LMICsalthough limited-suggests that outcomes for acutely unwell patients are poorer when compared with high-income countries (HICs).${ }^{12}$ Increasingly, the inability measures continuous information to evaluate routine care and empower stakeholders to identify priorities for improvement is acknowledged as an important missing link in health system infrastructure. $^{3}$

In HICs, national evaluation of outcomes, benchmarking of quality indicators and patient experience are driving care improvement and the way resources and services are delivered in acute care. ${ }^{4}$ In low-income and middle-income countries (LMICs), the lack of reliable facility level and national information has hampered attempts to continually evaluate the quality of care, hindered implementation of quality improvement initiatives and disempowered clinicians from identifying local research priorities. There has been little investment in health systems infrastructure or training for clinicians and administrators seeking to evaluate care in $\mathrm{LMICs}^{5}$ (figure 1). ${ }^{356} \mathrm{In}$ addition, systematic information pertaining to patient experience 


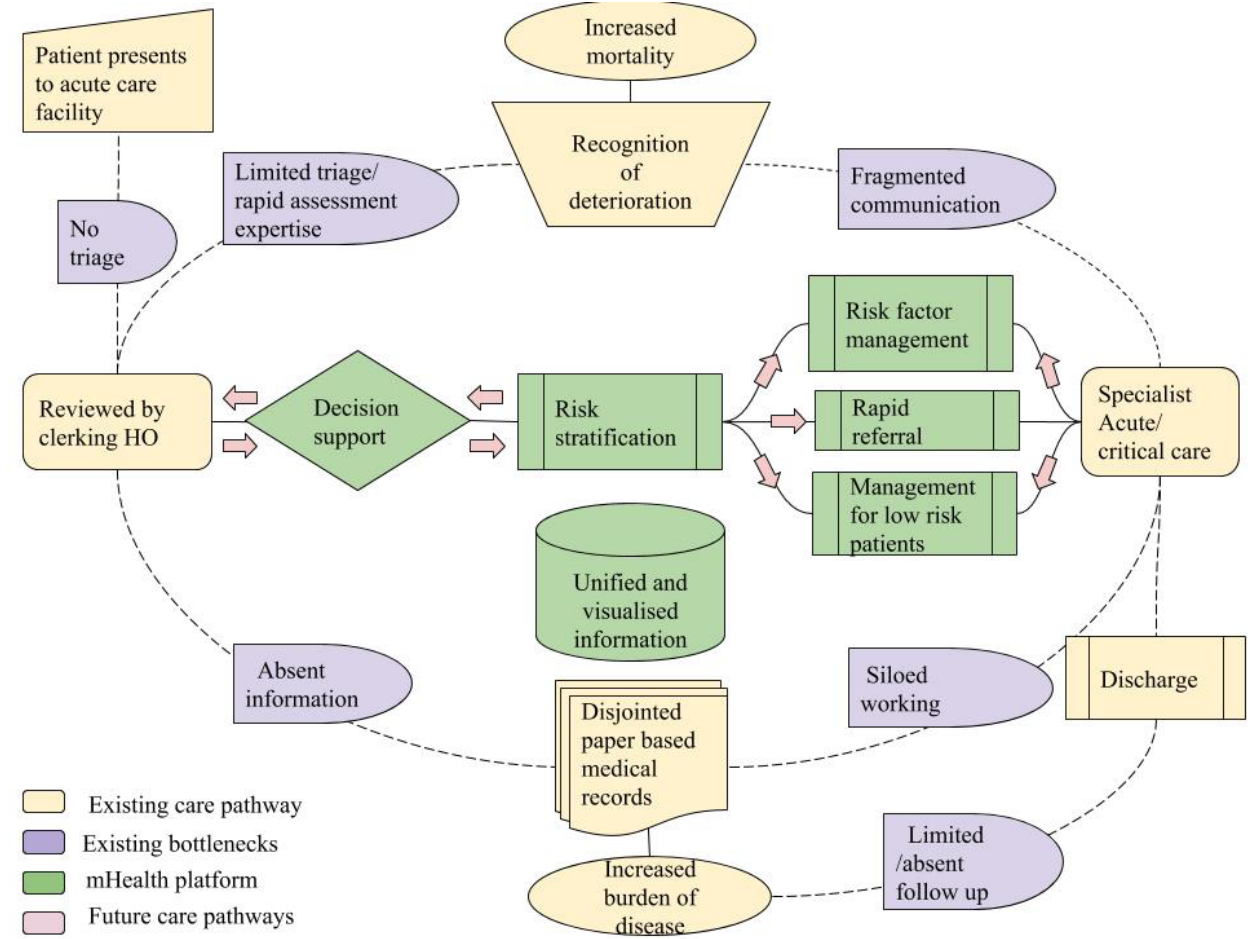

Figure 1 Existing pathways and bottlenecks for information flow and proposed enhanced systems following mHealth platform implementation for acute and critical care.

and recovery following acute or critical illness is virtually non-existent in LMIC settings. ${ }^{7-9}$

While there is much in the literature to recommend what data should be collected, ${ }^{7} 10^{11}$ there is limited practical advice or examples on how day-to-day clinical information can be successfully captured, especially in overstretched and under-resourced LMIC settings. ${ }^{1}$ It is noticeable in HIC settings that healthcare providers and researchers have increasingly turned towards technology and digital surveillance in order to achieve the breadth and saturation of continuous information needed to improve care. The worldwide digital boom in accessible mobile technology and internet connectivity over the last 5 years has been most pronounced in South Asia and regions of sub-Saharan Africa. ${ }^{12}$ Digital (mHealth) tools to connect remote communities, most notably in health promotion and primary disease prevention, have been shown to have an impact on patients' self-education and adherence to treatment. In contrast to these community settings, application of similar systems in acute and tertiary care in LMIC have been less well explored. ${ }^{9} 1314$ Instead, disparate, paper-based systems persist, with patient records remaining ununified as patients move through the healthcare system, not only delaying the delivery of clinical care but also hindering efforts by clinicians seeking to prognosticate, benchmark and improve care. ${ }^{15}$ Complex routes to admission, heterogeneous populations, diverse patient journeys and the need to synthesise often high volumes of clinical and laboratory information from multiple sources further hinder efforts to apply technology in the acute setting, especially in LMICs.
To be effectively adopted within health systems, such innovations must harmonise with existing workflows, empower users, minimise user risk, optimise use of existing resources and augment evidence-based clinical management. ${ }^{4912}$ The ability to integrate with existing or scaled national health information programmes is also essential if technologies are to be transferable with future traction beyond the existing infrastructure. These onerous requirements -often underestimated even in HIC- can seem insurmountable in resource-limited settings, where existing barriers to the adoption of such systems include cost, variable technology infrastructure and a perceived lack of value among busy clinicians. ${ }^{14}$ In addition, overburdened frontline staff are often unable to engage with such systems, perhaps due in part to the perceived enormity of behavioural change, task shift and burden of data capture that is potentially required. ${ }^{14}$ Furthermore, frontline clinicians also highlight the limited opportunities available to develop the necessary skills to heuristically evaluate care improvements in their healthcare facility. ${ }^{16}$

In this article, we draw from our experiences on designing, implementing and evaluating a clinician-led national digital mHealth information platform in acute care settings in Sri Lanka. We aim to share lessons on how successful engagement of clinicians in development and evaluation of the platform can help overcome the potential barriers to adoption described above. We also consider how this experience in Sri Lanka is now informing implementation and scaling of the platform in Pakistan, and how the methodology has potentially wider relevance for others seeking to address the information deficit for improving acute care in other LMICs. 


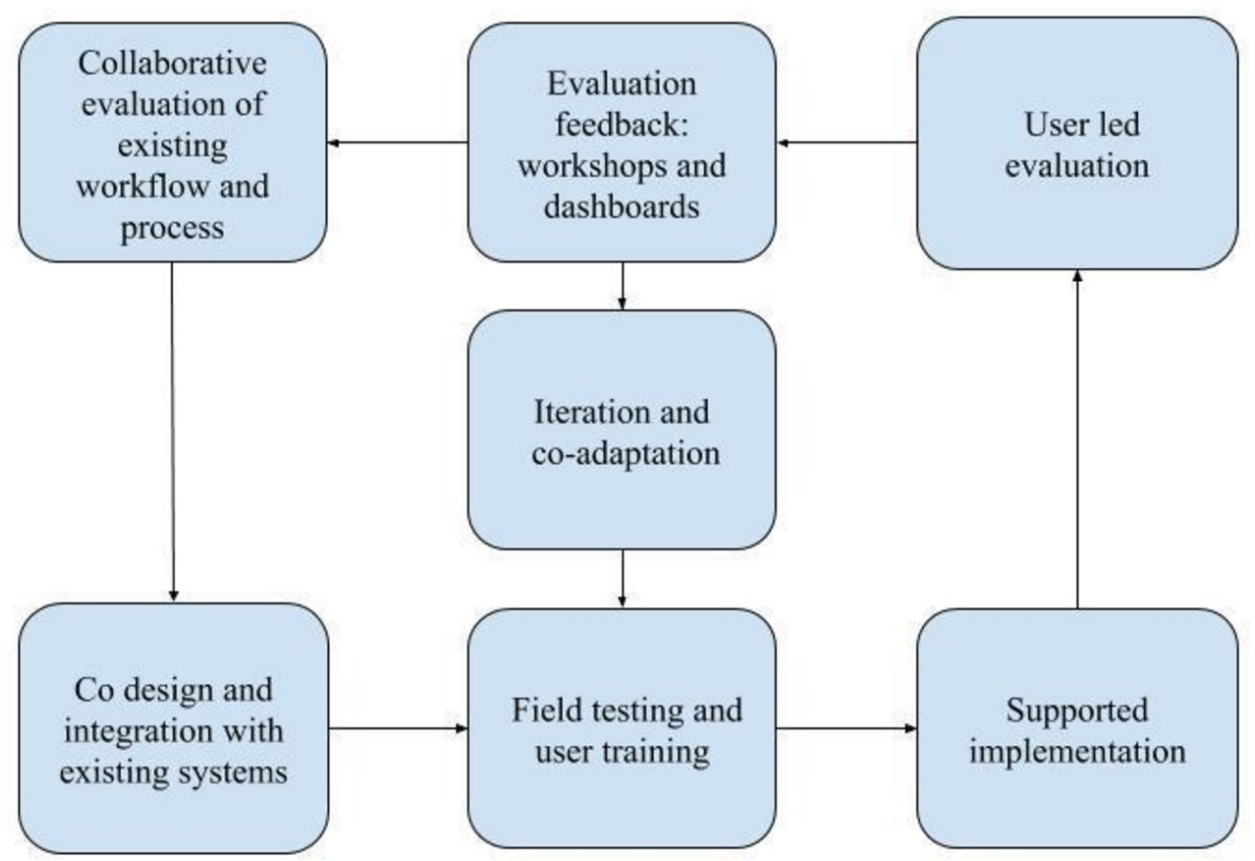

Figure 2 Cycle of implementation and co-evaluation.

\section{SETTING}

Nestled in South Asia, Sri Lanka has seen rapid economic, industrial growth and urbanisation in the last decade. ${ }^{17}$ Sri Lanka has outperformed neighbouring countries in public health and social welfare with high rates of literacy $(91 \%)$, the lowest maternal mortality and the presence of a robust public health network, coordinated through the Ministry of Health. ${ }^{3}$ The country is experiencing growth of commercial and services industry from a predominantly agricultural past. As in other LMICs, non-communicable disease burden is on the rise resulting in increasing demand on tertiary services, including but not

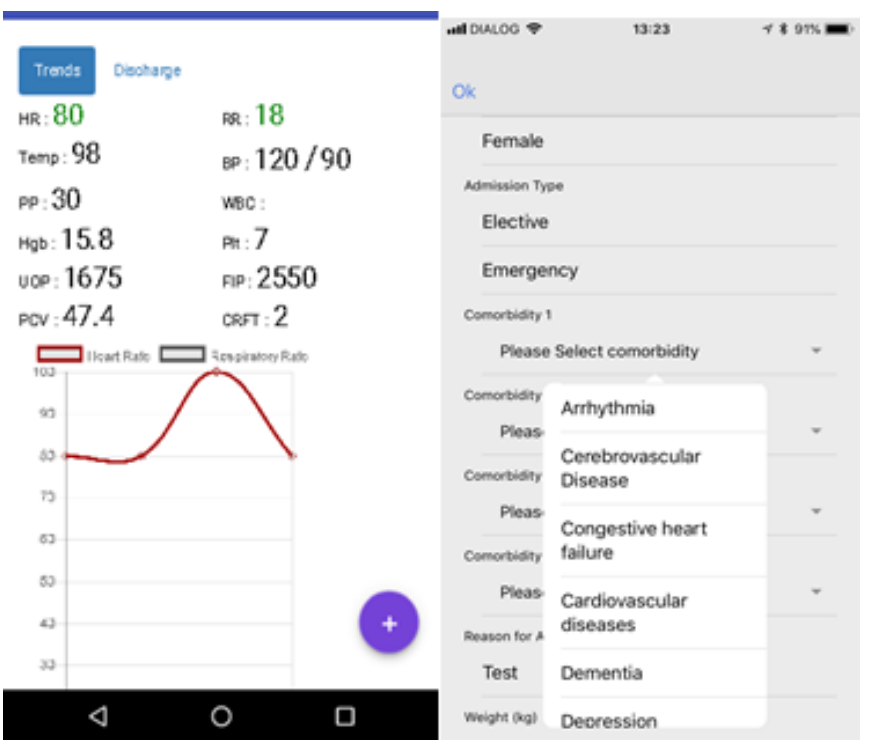

Figure 3 Network for Improving Critical care Skills Training user interface. limited to cardiovascular, surgical, obstetric, emergency and critical care. ${ }^{18}$

\section{APPROACH}

To address the deficits in granular information for both clinical care and for facility-level healthcare evaluation, an international not-for-profit collaborative consisting of clinical researchers, administrators and healthcare information technologists constructed a clinician-led, acute care digital mHealth platform (Platform for Reporting Outcomes, Epidemiology, Clinical Trends, and Surveillance). Motivated by an ambition to address the need for data-driven healthcare improvement that empowers users, the platform was developed using a cycle of implementation, co-evaluation and feedback. ${ }^{13}$ To support evaluation of adoption, qualitative enquiry founded in technology adoption frameworks were utilised to guide understanding of potential social and behavioural influences of the team. ${ }^{17}$

\section{Design}

Working directly in partnership with the Ministry of Health, clinicians and administrative end users, a minimal data set was derived based on previous work undertaken by the collaboration. ${ }^{719}$ This minimum data was purposefully narrow to enable coalesced deployment of the platform across acute care facilities including critical care, surgical and perioperative care and acute medicine. ${ }^{20}$ Diagnoses, procedures and comorbidities were described using commonly used, context-specific terminology, offering users words and phrases synonymous with existing practice. Inbuilt mapping to universally compatible coding (International Statistical Classification of 


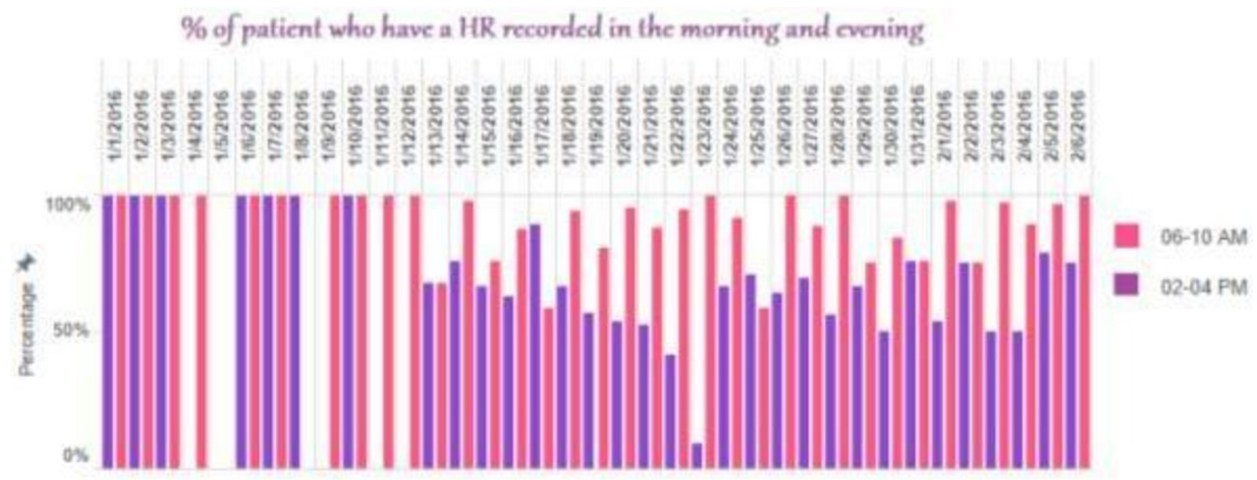

Figure 4 Quality dashboards in the ward setting help nurses improve completeness of vital sign reporting during adoption.

Diseases and Related Health Problems 10th Revision and Systematized Nomenclature of Medicine), ${ }^{21} 22$ facilitates research and increases the shareability of the information for future collaborative studies. Vital signs (eg, measure of mentation, respiratory rate, pulse, temperature, blood pressure), basic biochemical, haematological and microbiological measures were captured, with trends highlighting abnormal measures within the patient profile. Patient-reported measures of quality of life following discharge (EQ5D) ${ }^{23}$ were captured through the platform by a trained support officer using telephone follow-up.

The user interface is designed to be navigated as patients progress through their journey of care. For example, information pertaining to presentation and reasons for admission are visible on the clinician's home screen following login, and information can be edited throughout the patients treatment progression. Edits are stored sequentially ensuring an audit trail of information. Laboratory test and physiological observations can be added sequentially and are viewed in a single window, aiding treatment decision-making.

Offline functionality and visual alerts for connectivity and data synchronisation status within the platform helped minimise the challenges of intermittent internet connectivity and provides confirmation to users that information entry and upload was successful. This visual reassurance was an important step in building trust between users and the technology.

Co-designed desktop and tablet dashboards, using non-proprietary analytics software and a business analytics tool visualise trends for individual patients and aggregate groups tailored to reflect the user's priorities. For example, automated visual alerts were applied to
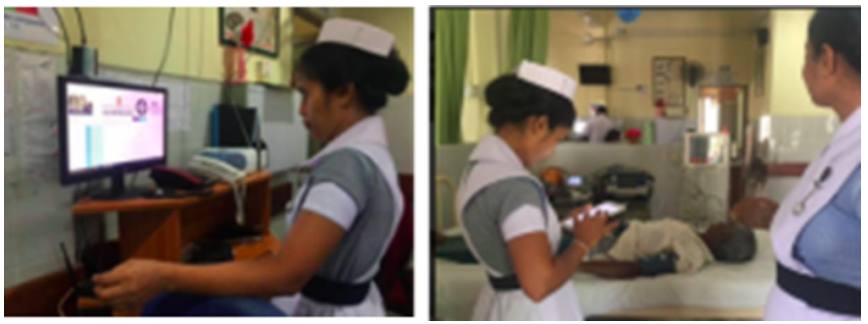

Figure 5 Acute care mHealth platform adopted in an inpatient setting in Sri Lanka. abnormal physiological measures, helping nurses and doctors identify potentially unwell patients. Simultaneously and in real time, admission, occupancy and information regarding length of stay were made available to hospital management and administrators seeking to optimise organisational aspects of care, staffing, elective surgeries and critical care bed capacity. This information is visible through desktop and mobile devices.

\section{Implementation}

The platform was implemented at the invitation of and in partnership with clinical teams, hospital administration teams, professional bodies (eg, professional colleges) and ministry departments. The collaborative drew on methods from the disciplines of improvement and behavioural science (human factors) and engineering to help identify drivers and barriers to adoption. ${ }^{17}{ }^{23}$ Facilitator-led focus groups were used to support early phase adoption alongside a traditional cycle of implementation and evaluation (plan, do, study, act) (figure 2).

Clinical setting champions (often senior nurses well placed to facilitate the behavioural change needed for adoption, given their central role in the daily organisation of patient care) were trained to lead implementation in their clinical area. They supported peer training and troubleshooted technical or task allocation challenges and challenges in the daily utilisation of the platform. A site coordinator made daily visits to the clinical settings during the initial implementation phase (2 weeks), liaising with the local champion and supporting the team with using the platforms features. Following initial implementation, day-to-day support was provided remotely via telephone to review progress, identify new barriers to sustainable use and identify processes of care where the platform may add value.

\section{EVALUATION Adoption}

The platform has been successfully adopted in 56 acute healthcare facilities (including 102 intensive care units) in Sri Lanka and supports the only national intensive care registry in South Asia (figure 3), which commenced in 2012 took 2 years to scale nationally. 
Scaling of the platform across acute care was driven by frontline stakeholder engagement and on an invitation basis. Seven sites dropped out during implementation, citing concerns regarding sharing of information. Recruitment remains active. Completeness of reporting of information is validated weekly by cross-referencing aggregate admission numbers and this is then reported through the visual dashboard and as part of a monthly individualised summary for each centre.

Clinician-led adoption, using locally driven cycles of implementation and qualitative interviews, enabled identification of drivers and acceptable solutions to key barriers; burden of data collection, integration into workflow and the potential for legal implications of transition from paper to digital documentation. Focus group discussion with frontline users (nurses and doctors) led to iterative adaptation of the platform. Adaptation included development of aggregate reports on completeness of vital sign reporting which provided positive reinforcement and direct feedback during the adoption phase (figure 4 ). ${ }^{84}$

Focus groups including nurses, doctors and allied healthcare professionals elicited potential barriers such as time taken to enter and find information at critical steps (admission, ward round and discharge) in patient care. Feedback from users led to the addition of an editable and auditable 'reason for admission' field and the 'diagnosis field' mapped to international classification being placed in the discharge section of the user interface. Trends in physiological variables, common presentations and patient groups at high risk of adverse events were highlighted, facilitating treatment evaluation and providing objective data for ward rounds, empowering the nurses role in monitoring of unwell patients and supporting communication between team members.

Mobile accessibility of information has been an essential feature of the platforms usefulness in the acute-care setting enabling acute care teams a reliable mechanism to record, view and communicate information as the patient and the clinician move from emergency department, ward, critical care, surgery and clinic during daily care (figure 5 ).

Features for saving information as PDF for printing were added in response to user feedback, to enable information sharing with patients and community services on discharge. Printing options also helped reduce anxiety over the perceived legal superiority of paper over electronic records and integrating digital and hand written information in paper-based medical records. The platform was further used to facilitate training and governance meetings, providing clinicians with accessible information to review care processes, such as recognition of sepsis postoperatively, and time from referral to intervention in acute cardiac care, and to direct learning regarding clinical presentation and diagnosis. Furthermore, nurses reported an improved nurse and patient relationship, giving patients a greater feeling of safety by having information regarding their care visible.

These adaptation based on user feedback reflect the dynamic nature of acute care workflow which necessitates information to be accessible to healthcare workers and patients throughout their continuum of care. As barriers to adoption have been addressed in an iterative manner, implementation in new sites in both Sri Lanka has become increasingly efficient with little or no new barriers to adoption in the clinical settings arising. This may suggest that the barriers and facilitators to adoption of technology in these settings are somewhat universal and the solutions potentially transferable.

\section{Utilisation}

\section{Service evaluation}

The platform has assisted clinicians in the care of over 100000 patient episodes, from critical care admission, through to outcomes at 30 days following discharge. ${ }^{24}$ The digital intensive care unit (ICU) platform has increased availability of variables for benchmarking acuity of admissions and supported the validation of prognostic models. ${ }^{11} 192025$ The platform has also facilitated participation in international multisite observational studies on ventilation management. ${ }^{26}$ Prior studies attempting to answer similar questions in this setting have highlighted challenges of lack of information, missing outcomes and small data sets. The platforms ability to increase availability of routine clinical information, address known potential barriers to technology adoption and subsequent evaluation of clinical care is an important step forward in establishing health systems in LMICs equipped to evaluate and improve care.

\section{Service delivery}

Real-time aggregate information generated by the platform supports a 24-hour national bed availability system which has assisted clinicians locate ICU beds for over 3700 patients. ${ }^{1124}$ The system has highlighted barriers to successful patient transfer including geographic distance of the available bed from the referral centre and clinical instability of the patient. Nurses, who are often early adopters of the platform report value in access to information on ward activity, acuity and staff to patient ratios, which, displayed on desktop dashboards have helped them overcome existing burden of collecting and reporting this information manually as part of existing monthly organisational reports.

\section{Decision support in acute clinical care}

In the acute care setting, the adapted mobile version of the digital platform has empowered nurses and doctors to adopt digital vital sign reporting (500 000 observations) in busy ward settings. ${ }^{28}$ Adaptation of an early warning score has helped identify over 4800 deteriorating ward patients by flagging patients who have deranged physiology on admission, for whom 
increased frequency of vital signs monitoring may be beneficial. ${ }^{26-28}$

\section{Health system research}

The platform has enabled national and international research in prognostic modelling in acute care (including for sepsis), the external validation of severity of illness models and related risk stratification tools. ${ }^{19} 202529$ Risk stratification of surgical patients, incidence of postoperative complications, description of quality of life for patients following critical illness and surgery and patient experiences and satisfaction following critical care admission have also been evaluated. ${ }^{30}$ Focus group discussion with frontline users during implementation has engaged stakeholders in further care evaluation. Using the platform implementation as a lens, clinical teams are gaining insights into the existing processes in patients care. For example, in acute care wards, the dashboards visual display of the location of patients with abnormal vital signs is prompting nurses to consider reorganisation of the location of unwell patients within the ward to an area designated for closer observation or with the availability of continuous monitoring.

\section{Remaining Challenges}

Perhaps, the greatest challenges remain in understanding the more hidden barriers that prevent clinicians from actively using information captured through such platforms to drive change. Recent literature suggests that such impediments may extend beyond knowledge, opportunity and resource. ${ }^{31}$ Further exploration of healthcare worker perceptions in resource-limited settings is needed. Similarly, the disparity that exists between countries in support for clinicians seeking to undertake research and training in these domains needs to be addressed.

In addition, further work is needed to engage researchers, clinicians, administrators, developers and the public regarding security, safety and risks of digital information. While all acknowledge the flaws of existing paper systems and the negligible security of the information that dwells dormant in healthcare facilities internationally, there remains anxiety over the potential for misuse of digital information, especially in LMIC. Perhaps, empowering patients to be the gatekeepers and equal stakeholders in their information and in the care it informs, as pioneered with patient-reported outcomes in surgical care, offers a way forward.

\section{Transferability}

The platform is being implemented across 18 intensive care units in four provinces in Pakistan; the collaboration has shared technology, approach to implementation and solutions to the barriers to adoption in a South-to-South partnership. ${ }^{32}$ While Sri Lanka has a predominantly centralised government-led health system, governance in Pakistan is devolved to regional organisations with a higher proportion of privately funded facilities. The approach to stakeholder engagement, frontline stakeholder driven collaboration with professional bodies, ministry organisation already identified as key stakeholders in Sri Lanka, is proving effective in implementation in Pakistan.

Implementation challenges in Pakistan have so far been minimal compared with the original registry rollout in Sri Lanka. Implementation in Sri Lanka starting back in 2012 required installing internet and landlines in each ICU, whereas IT infrastructure in Pakistan was comparatively well developed already, reflecting the significant growth in internet and digital connectivity in the region. The user-friendly mobile application-based platform has been easily introduced without the need for extensive end-user training or installation of software. The registry offline functionality has been successful in overcoming interrupted internet connectivity, which remains a challenge in the region. Sri Lanka is in the process of developing and implementing a national digital health information system. This system is currently under pilot implementation in selected facilities. The PROTECTS platforms ability to harmonise with existing systems has enabled successful co-implementation to date and we look forward to collaborating on future integration (online supplementary file).

\section{NEXT STEPS}

A mixed-methods evaluation of critical care outcomes including measures of quality of life and patient-reported outcomes is underway in Sri Lanka. 'Work is currently underway within the collaboration to identify priorities for decision support tools incorporating prognostic models validated and developed from the information captured through the platform'.

Mobile interfaces are being developed for patient-reported information giving patient greater opportunity to participate in the decision regarding their care and guiding patient-led health service improvement. The dashboards are providing a real-time feedback to facilitate setting-specific research focused on evaluating and improving the quality of care, antibiotic use and time to referral for patients with myocardial infarction. ${ }^{19} 202630$

Funding is being sought to scale the platform in Pakistan and to partner with other LMIC in sub-Saharan Africa, who are keen to collaborate by employing both the technology and methodology described here.

\section{CONCLUSION}

Adoption of digital mHealth platforms to support acute care in South Asia is feasible. Such platforms can help close the gap in availability of continuous facility level and national information needed for service evaluation and research. A collaborative approach engaging clinicians, researchers and healthcare informaticians offers a pragmatic solution to overcoming barriers to adoption, 
disruption to workflow and user engagement. Digital platforms can support evaluation of existing care at local and national levels and empower clinicians working in LMIC to participate in international research. Greater investment in successful methodologies for health system improvement will improve their penetration in LMIC health systems. Accessible opportunities for clinicians and patients in resource-limited settings to set the agenda for research better and directly use output from digital platforms are required.

Contributors This practice paper describes the experiences and insights of a multidisciplinary collaborative. Each author is a key stakeholder in the collaborative and represents a wider network. All made significant contribution to the learning described. $A B$ and $R H$ were responsible for manuscript preparation.

Funding This study was part funded by National Science Foundation, Sri Lanka and by NICST.

Competing interests None declared.

Patient consent for publication Not required.

Provenance and peer review Not commissioned; externally peer reviewed.

Data sharing statement No additional data are available.

Open access This is an open access article distributed in accordance with the Creative Commons Attribution Non Commercial (CC BY-NC 4.0) license, which permits others to distribute, remix, adapt, build upon this work non-commercially, and license their derivative works on different terms, provided the original work is properly cited, appropriate credit is given, any changes made indicated, and the use is non-commercial. See: http://creativecommons.org/licenses/by-nc/4.0/.

\section{REFERENCES}

1. Travis P, Bennett S, Haines A, et al. Overcoming health-systems constraints to achieve the millennium development goals. The Lancet 2004;364:900-6.

2. Biccard BM, Madiba TE, Kluyts H-L, et al. Perioperative patient outcomes in the African Surgical Outcomes Study: a 7-day prospective observational cohort study. The Lancet 2018;391:1589-98.

3. Lopez AD, Mathers CD, Ezzati M, et al. Global and regional burden of disease and risk factors, 2001: systematic analysis of population health data. The Lancet 2006;367:1747-57.

4. Ridde V. Need for more and better implementation science in global health. BMJ Glob Health 2016;1:e000115.

5. Dondorp AM, lyer SS, Schultz MJ. Critical care in resource-restricted settings. JAMA 2016;315:753-4.

6. Abimbola S. The information problem in global health. BMJ Glob Health 2016;1:e900001.

7. Haniffa R, De Silva AP, Iddagoda S, et al. A cross-sectional survey of critical care services in Sri Lanka: a lower middle-income country. $J$ Crit Care 2014;29:764-8.

8. Beane A, Padeniya A, De Silva AP, et al. Closing the theory to practice gap for newly qualified doctors: evaluation of a peerdelivered practical skills training course for newly qualified doctors in preparation for clinical practice. Postgrad Med J 2017;93:592-6.

9. De Silva AP, Harischandra PL, Beane A, et al. A data platform to improve rabies prevention, Sri Lanka. Bull World Health Organ 2017:95:646-51.

10. De Silva A, Sujeewa J, De Silva N. A retrospective study of physiological observation reporting practices and the recognition, response, and outcomes following cardiopulmonary arrest in a Lower- middle Income Country. Indian J Crit Care Med 2013;21:343-5

11. Beane A, De Silva AP, De Silva N, et al. Evaluation of the feasibility and performance of early warning scores to identify patients at risk of adverse outcomes in a low-middle income country setting. BMJ Open 2018;8:e019387.

12. Chalkidou K, Li R, Culyer AJ, et al. Health Technology assessment: global advocacy and local realities comment on "priority setting for universal health coverage: we need evidence-informed deliberative processes, not just more evidence on cost-effectiveness". Int J Health Policy Manag 2017;6:233-6.

13. Beane A, Athapattu PL, Dondorp AM, et al. Commentary: challenges and priorities for pediatric critical care clinician-researchers in lowand middle-income countries. Front Pediatr 2018;6:38.

14. Matsumoto D. Culture, context, and behavior. $J$ Pers 2007;75:1285-320.

15. Dare AJ, Onajin-Obembe B, Makasa EM. A snapshot of surgical outcomes and needs in Africa. The Lancet 2018;391:1553-4.

16. Haniffa R, Lubell Y, Cooper BS, et al. Impact of a structured ICU training programme in resource-limited settings in Asia. PLoS One 2017; 12:e0173483.

17. Venkatesh V, Morris MG, Davis GB, et al. User acceptance of information technology: toward a unified view. MIS Quarterly 2003;27:425-78.

18. Engelgau M, Okamoto K, Navaratne KV, et al. Prevention and control of selected chronic NCDs in Sri Lanka, 2010.

19. Haniffa R, Pubudu De Silva A, Weerathunga P, et al. Applicability of the APACHE II model to a lower middle income country. $J$ Crit Care 2017:42:178-83.

20. Haniffa R, Mukaka M, Munasinghe SB, et al. Simplified prognostic model for critically ill patients in resource limited settings in South Asia. Crit Care 2017;21:250.

21. Quan H, Sundararajan V, Halfon P, et al. Coding algorithms for defining comorbidities in ICD-9-CM and ICD-10 administrative data. Med Care 2005;43:1130-9.

22. Snomed CT. International health terminology standards development organization, 2015

23. Rabin R, de Charro F, Charro Fde. EQ-5D: a measure of health status from the EuroQol Group. Ann Med 2001;33:337-43.

24. Stephens T, De Silva AP, Beane A, et al. Capacity building for critical care training delivery: development and evaluation of the Network for Improving Critical care Skills Training (NICST) programme in Sri Lanka. Intensive Crit Care Nurs 2017;39:28-36.

25. Beane A, De Silva A, Munasinghe S. Comparison of quick Sequentia Organ Failure Assessment (qSOFA) and modified Systemic Inflammatory Response Syndrome (mSIRS) criteria in a lower middle income setting. J Acute Med 2017;7:141-8.

26. Pisani L, Algera AG, Serpa Neto A, et al. PRactice of VENTilation in Middle-Income Countries (PRoVENT-iMIC): rationale and protocol for a prospective international multicentre observational study in intensive care units in Asia. BMJ Open 2018;8:e020841.

27. Rathnayake S. Smart content for smart people best practices of Sri Lankan e-Content and applications of 2014, 2014.

28. Haniffa R, De Silva A, Beane A, et al. A cloud-based national critical care registry - a South Asian LMICs data platform. Rev Bras Ter Intensiva. In Press 2018.

29. Rudd KE, Seymour CW, Aluisio AR, et al. Association of the Quick Sequential (Sepsis-Related) Organ Failure Assessment (qSOFA) Score with excess hospital mortality in adults with suspected infection in low- and middle-income countries. JAMA 2018;319:2202-11.

30. Beane $A$, Wagstaff $D$, Abayadeera $A$, et al. Surgical surveillance in resource-poor settings. The Lancet 2018;391:1571.

31. Conradie A, Forget DP, Biccard BM. Barriers to clinical research in Africa: a quantitative and qualitative survey of clinical researchers in 27 African countries. BJA. In Press 2018.

32. Hashmi M, Beane A, Memon M, et al. Pakistan Registry of Intensive Care (PRICE): expanding a lower middle-income, clinician-designed critical care registry in South Asia. J Intensive Care Soc. In Press 2018. 